\title{
ON PULSATING AND CELLULAR FORMS OF HYDRODYNAMIC INSTABILITY IN LIQUID-PROPELLANT COMBUSTION
}

\author{
STEPHEN B. MARGOLIS \\ Combustion Research Facility, MS 9052 \\ Sandia National Laboratories \\ Livernore, CA 94551-0969, USA
}

\begin{abstract}
An extended Landau-Levich model of liquid-propellant combustion, one that allows for a local dependence of the burning rate on the (gas) pressure at the liquid-gas interface, exhibits not only the classical hydrodynamic cellular instability attributed to Landau but also a pulsating hydrodynamic instability associated with sufficiently negative pressure sensitivities. Exploiting the realistic limit of small values of the gas-to-liquid density ratio $\rho$, analytical formulas for both neutral stability boundaries may be obtained by expanding all quantities in appropriate powers of $\rho$ in each of three distinguished wave-number regimes. In particular, composite analytical expressions are derived for the neutral stability boundaries $A_{p}(k)$, where $A_{p}$ is the pressure sensitivity of the burning rate and $k$ is the wave number of the disturbance. For the cellular boundary, the results demonstrate explicitly the stabilizing effect of gravity on long-wave disturbances, the stabilizing effect of viscosity (both liquid and gas) and surface tension on short-wave perturbations, and the instability associated with intermediate wave numbers for negative values of $A_{\mu}$, which is characteristic of many hydroxylammonium nitrate-based liquid propellants over certain pressure ranges. In contrast, the pulsating hydrodynamic stability boundary is insensitive to gravitational and surface-tension effects but is more sensitive to the effects of liquid viscosity because, for typical nonzero values of the latter, the pulsating boundary decreases to larger negative values of $A_{p}$, as $k$ increases through $\mathrm{O}(1)$ values. Thus, liquid-propellant combustion is predicted to be stable (that is, steady and planar) only for a range of negative pressure sensitivities that lie below the cellular boundary that exists for sufficiently small negative values of $A_{i}$, and above the pulsating boundary that exists for larger negative values of this parameter.
\end{abstract}

\section{Introduction}

The stability of liquid-propellant deflagration is a fundamental problem that was first treated by Landau in a classical study [1] that introduced the concept of hydrodynamic instability in a combustion context. Referred to as the "slow combustion of liquids," that analysis is most applicable to certain realistic, limiting cases in which combustion may be approximated by an overall reaction at the liquidgas interface. For example, the gas flame may occur under near-breakaway conditions, exerting little thermal or hydrodynamic influence on the burning propellant; or distributed combustion may only occur in an intrusive regime such that the reaction zone lies closer to the liquid-gas interface than the length scale of any disturbance of interest; or the liquid propellant may simply undergo exothermic decomposition at the surface without any significant distributed combustion, such as appears to occur in some types of hydroxylammonium nitrate (HAN)based liquid propellants at low pressures [2]. The results of Landau's study, along with a subsequent paper by Levich [3] that replaced the effects due to surface tension in the earlier study with those due to (liquid) viscosity, have been widely quoted and offer much in terms of physical insight into the nature of this type of instability, which, as in the case of gaseous combustion, is associated with the density change across the reaction front. However, because these models assumed a constant normal burning rate, it has proven useful to improve upon them by incorporating a more realistic coupling of the normal propagation speed with the local pressure and temperature fields, thereby allowing for a locally varying burning rate $[4,5]$. One result that has emerged from this generalization is that, in addition to the classical Landau (cellular) type of instability, the models now predict a pulsating hydrodynamic instability as well. The latter arises specifically from the local pressure coupling and thus may be physically achievable because the mass buming rate of many propellants has been shown empirically to correlate well with pressure. Thermal coupling, on the other hand, 
introduces additional thermal/diffusive instabilities [5] that will not be considered here. In the present work, we shall consider both types of hydrodynamic instabilities, but particular focus will be placed on the pulsating stability boundary, because this type of instability is absent from the earlier models that neglected the pressure coupling previously indicated. As in a companion study that focused on the classical (cellular) hydrodynamic instability [6], we develop a formal asymptotic theory by considering the realistic limiting case in which the gas-to-liquid density ratio $\rho$ is small.

\section{Mathematical Model}

The governing hydrodynamic equations consist of mass and momentum on èither side of the gas-liquid interface, supplemented by a pressure-dependent burning-rate law and associated continuity and jump conditions across the interface [4-6]. Thus, it is assumed, as in the classical models, that there is no distributed reaction in either the liquid or gas phases but that there exists either a pyrolysis reaction or an exothermic decomposition at the liquid-gas interface that depends on the local pressure. For simplicity, it is assumed that within the liquid and gas phases separately, the density and other fluid properties are constants, with appropriate jumps across the phase boundary. The nondimensional location of the latter is denoted by $x_{3}=\Phi_{s}\left(x_{1}, x_{2}, t\right)$, where the adopted coordinate system is fixed with respect to the stationary liquid at $x_{3}=-\infty$. Then, in the moving coordinate system $x=x_{1}, y=x_{2}, z=x_{3}$ $\Phi_{s}\left(x_{1}, x_{2}, t\right)$, in terms of which the liquid-gas interface always lies at $z=0$, the complete nondimensional formulation of the problem in the absence of thermal coupling is given by

$$
\begin{gathered}
\nabla \cdot \mathbf{v}=0, \quad z \neq 0 \\
\frac{\partial \mathbf{v}}{\partial t}-\frac{\partial \Phi_{s}}{\partial t} \frac{\partial \mathbf{v}}{\partial z}+(\mathbf{v} \cdot \nabla) \mathbf{v}=\left(0,0,-F r^{-1}\right) \\
-\left\{\begin{array}{c}
1 \\
\rho^{-1}
\end{array}\right\} \nabla p+\left\{\begin{array}{c}
\operatorname{Pr}_{l} \\
\lambda \operatorname{Pr}_{g}
\end{array}\right\} \nabla^{2} \mathbf{v}, \quad z \lessgtr 0
\end{gathered}
$$

subject to $v=0$ at $z=-\infty$ and the interface conditions

$$
\begin{aligned}
& \hat{\mathbf{n}}_{\mathbf{s}} \times \mathbf{v}_{-}=\hat{\mathbf{n}}_{\mathrm{s}} \times \mathbf{v}_{+} \\
& \hat{\mathbf{n}}_{\mathbf{s}} \cdot\left(\mathbf{v}_{-}-\rho \mathbf{v}_{+}\right)=(1-\rho) S\left(\Phi_{s}\right) \frac{\partial \Phi_{s}}{\partial t} \\
& \hat{\mathbf{n}}_{\mathbf{s}} \cdot \mathbf{v}_{-}-S\left(\Phi_{s}\right) \frac{\partial \Phi_{s}}{\partial t}=A\left(p_{+}\right)
\end{aligned}
$$

$$
\begin{aligned}
p_{-} & -p_{+}=\hat{\mathbf{n}}_{s} \cdot\left[\rho \mathbf{v}_{+}\left(\hat{\mathbf{n}}_{s} \cdot \mathbf{v}_{+}\right)-\mathbf{v}_{-}\left(\hat{\mathbf{n}}_{s} \cdot \mathbf{v}_{-}\right)\right. \\
& \left.-\rho \lambda P r_{s} \mathbf{e}_{+} \cdot \hat{\mathbf{n}}_{s}+\operatorname{Pr} \mathbf{e}_{-} \cdot \hat{\mathbf{n}}_{s}\right] \\
& +\hat{\mathbf{n}}_{s} \cdot\left(\mathbf{v}_{-}-\rho \mathbf{v}_{+}\right) S\left(\Phi_{s}\right) \frac{\partial \Phi_{s}}{\partial t} \\
& -\gamma S^{3}\left(\Phi_{s}\right)\left\{\frac{\partial^{2} \Phi_{s}}{\partial x^{2}}\left[1+\left(\frac{\partial \Phi_{s}}{\partial y}\right)^{2}\right]\right. \\
& \left.+\frac{\partial^{2} \Phi_{s}}{\partial y^{2}}\left[1+\left(\frac{\partial \Phi_{s}}{\partial x}\right)^{2}\right]-2 \frac{\partial \Phi_{s}}{\partial x} \frac{\partial \Phi_{s}}{\partial y} \frac{\partial^{2} \Phi_{s}}{\partial x \partial y}\right\} \\
\hat{\mathbf{n}}_{\mathbf{s}} & \times\left[\rho \mathbf{v}_{+}\left(\hat{\mathbf{n}}_{\mathrm{s}} \cdot \mathbf{v}_{+}\right)-\mathbf{v}_{-}\left(\hat{\mathbf{n}}_{s} \cdot \mathbf{v}_{-}\right)\right. \\
& \left.+\left(\mathbf{v}_{-}-\rho \mathbf{v}_{+}\right) S\left(\Phi_{s}\right) \frac{\partial \Phi_{s}}{\partial t}\right] \\
& =\hat{\mathbf{n}}_{\mathbf{s}} \times\left(\rho \lambda \operatorname{Pr}_{S} \mathbf{e}_{+} \cdot \hat{\mathbf{n}}_{s}-\operatorname{Pr}_{l} \mathbf{e}_{-} \cdot \hat{\mathbf{n}}_{s}\right)
\end{aligned}
$$

where the latter represent continuity of transverse velocity components (no slip), conservation of (normal) mass flux, the mass burning-rate law, and conservation of normal and transverse components of momentum flux, respectively. Here, $\mathbf{v}$ and $p$ denote velocity [with respect to the original $\left(x_{1}, x_{2}, x_{3}\right)$ coordinate system] and pressure, and the \pm subscripts denote evaluation at $z=0^{ \pm}$. The parameters $P r_{l}$ and $\mathrm{Pr}_{\mathrm{g}}$ denote the liquid and gas-phase Prandtl numbers, $\rho$ and $\lambda$ are the gas-to-liquid density and thermal diffusivity ratios, $F r$ is the Froude number, $\mathbf{e}$ is the rate-of-strain tensor, and $\gamma$ is the surface-tension coefficient. All of these quantities are defined in terms of their dimensional counterparts in the nomenclature. In addition, the factor $S\left(\Phi_{s}\right)$ and the unit normal $\hat{\mathbf{n}}_{s}$ are given by $S\left(\Phi_{s}\right)=\left[1+\left(\partial \Phi_{s} / \partial x\right)^{2}\right.$ $\left.+\left(\partial \Phi_{s} / \partial y\right)^{2}\right]^{-1 / 2}$ and $\hat{\mathbf{n}}_{\mathrm{s}}=\left(-\partial \Phi_{s} / \partial x,-\partial \Phi_{s} / \partial y\right.$, 1) $S\left(\Phi_{s}\right)$, while the expressions for the gradient operator $\nabla$ and the Laplacian $\nabla^{2}$ in the moving coordinate system are given by $\nabla=[\partial / \partial x-$ $\left.\left(\partial \Phi_{s} / \partial x\right) \partial / \partial z, \partial / \partial y-\left(\partial \Phi_{s} / \partial y\right) \partial / \partial z, \partial / \partial z\right]$ and $\nabla^{2}=\partial^{2} / \partial x^{2}+\partial^{2} / \partial y^{2}+\left[1+\left(\partial \Phi_{s} / \partial x\right)^{2}+\right.$ $\left.\left(\partial \Phi_{s} / \partial y\right)^{2}\right] \partial^{2} / \partial z^{2}-2\left(\partial \Phi_{s} / \partial x\right) \partial^{2} / \partial x \partial z$ $2\left(\partial \Phi_{s} / \partial y\right) \partial^{2} / \partial y \partial z-\left(\partial^{2} \Phi_{s} / \partial x^{2}+\partial^{2} \Phi_{s} / \partial y^{2}\right) \partial / \partial z$. We remark that the factor multiplying $\gamma$ in equation 4 is the curvature $-\nabla \cdot \hat{\mathbf{n}}$, and note that $\rho \lambda \operatorname{Pr}_{\mathrm{g}}=\mu P r_{l}$, where $\mu$ is the gas-to-liquid viscosity ratio.

Finally, we observe that the burning rate $A(p)$ in the last of equations 3 is assumed, in accordance with numerous experimental correlations for both solid and liquid propellants [7], to depend on the local gas pressure at the interface, where $A$ is normalized to unity for the case of steady, planar burning. Indeed, in the linear stability analysis that follows, the pressure sensitivity $A_{p}=\partial A /\left.\partial p\right|_{p=0}$ (where, as indicated in equation 6 in the following, $p=0$ is the unperturbed gas pressure at $z=0^{+}$) of the local burning 
rate will emerge as an important parameter. In fact, $A_{p}$ is the only information about $A(p)$ that will appear explicitly, although in a nonlinear stability analysis, more detailed information in the form of higher derivatives would enter into the calculations (cf. Ref. [8]). We remark that, depending on the particular propellant, $A_{p}$ is not necessarily positive and may in fact take on zero and negative values over certain pressure ranges $[2,7]$.

A nontrivial basic solution to the foregoing problem, corresponding to the special case of a steady, planar deflagration, is given by

$$
\begin{aligned}
\Phi_{s}^{0} & =-t, \quad v^{0}=\left(0,0, v^{0}\right) \\
v^{0} & = \begin{cases}0 & z<0, \\
\rho^{-1}-1, & z>0\end{cases} \\
p^{0}(z) & = \begin{cases}-F r^{-1} z+\rho^{-1}-1, & z<0 \\
-\rho F r^{-1} z, & z>0\end{cases}
\end{aligned}
$$

The linear stability analysis of this solution now proceeds in a standard fashion. However, owing to the significant number of parameters, a complete analysis of the resulting dispersion relation is quite complex, and we follow our previous approach [6] by restricting further consideration to the realistic parameter regime $\rho \ll 1, \mu \ll 1$ and, in the case of microgravity, $\mathrm{Fr}^{-1} \ll 1$. In contrast, the earlier classical studies only considered special limiting cases and/or assumptions. Thus, in the study due to Landau [1], viscosity was neglected and the effects of gravity (assumed to act normal to the undisturbed planar interface in the direction of the unburned liquid) and surface tension were shown to be stabilizing, leading to a criterion for the absolute stability for steady, planar deflagration of the form (in our nondimensional notation) $4 \gamma \mathrm{Fr}^{-1} \rho^{2} /(1-\rho)>1$. In the study due to Levich [3], surface tension was neglected, but the effects due to the viscosity of the liquid were included, leading to the absolute stability criterion $\mathrm{Fr}^{-1} \operatorname{Pr}_{1}(3 \rho)^{3 / 2}>1$. Thus, these two studies, both of which assumed a constant normal burning rate $(A=1)$, demonstrated that sufficiently large values of either viscosity or surface tension, when coupled with the effects due to gravity, may render steady, planar deflagration stable to hydrodynamic disturbances. In our recent study [6], these results were synthesized and extended to the more realistic case of a nonconstant burning rate (i.e., $A_{p} \neq 0$ ) in the limiting parameter regime identified earlier. In the present work, we summarize these results for the classical cellular boundary and use the resulting scalings to derive an expression for the pulsating hydrodynamic stability boundary that arises from the pressure dependence of the local burning rate.

\section{Linear Stability Problem}

With respect to the basic solution, equation 6 , the perturbation quantities $\phi_{s}(x, y, t), \mathbf{u}(x, y, z, t)$, and $\zeta(x, y, z, t)$ are defined as $\Phi_{s}=\Phi_{s}^{\prime \prime}(t)+\phi_{s}, \mathbf{v}=$ $v^{\prime \prime}(z)+\mathbf{u}$, and $p=p^{\prime \prime}(z)+\zeta$, respectively. Substituting these definitions into the nonlinear model defined earlier and linearizing about the basic solution, the perturbation problem becomes

$$
\begin{gathered}
\frac{\partial u_{1}}{\partial x}+\frac{\partial u_{2}}{\partial y}+\frac{\partial u_{3}}{\partial z}=0, \quad z \neq 0 \\
\left\{\begin{array}{l}
1 \\
\rho
\end{array}\right\} \frac{\partial \mathbf{u}}{\partial t}+\frac{\partial \mathbf{u}}{\partial z}=-\left(\frac{\partial \zeta}{\partial x}+\left\{\begin{array}{l}
1 \\
\rho
\end{array}\right\} F r^{-1} \frac{\partial \phi_{s}}{\partial x}\right. \\
\left.\frac{\partial \zeta}{\partial y}+\left\{\begin{array}{l}
1 \\
\rho
\end{array}\right\} F r^{-1} \frac{\partial \phi_{s}}{\partial y}, \frac{\partial \zeta}{\partial z}\right) \\
+\left\{\begin{array}{c}
\operatorname{Pr}_{l} \\
\rho \lambda P r_{g}
\end{array}\right\}\left(\frac{\partial^{2} \mathbf{u}}{\partial x^{2}}+\frac{\partial^{2} \mathbf{u}}{\partial y^{2}}+\frac{\partial^{2} \mathbf{u}}{\partial z^{2}}\right), \quad z \lessgtr 0
\end{gathered}
$$

subject to $\mathbf{u}=0$ at $z=-\infty$ and

$$
\begin{aligned}
& \left.u_{1}\right|_{z=0^{-}}-u_{1} l_{z-0^{+}}=\left(\rho^{-1}-1\right) \frac{\partial \phi_{s}}{\partial x} \\
& \left.u_{2}\right|_{z=0^{-}}-\left.u_{2}\right|_{z=0^{+}}=\left(\rho^{-1}-1\right) \frac{\partial \phi_{s}}{\partial y} \\
& \left.u_{3}\right|_{z=0^{-}}-\left.\rho u_{3}\right|_{z=0^{+}}=(1-\rho) \frac{\partial \phi_{s}}{\partial t} \\
& \left.u_{3}\right|_{z=0^{-}}-\frac{\partial \phi_{s}}{\partial t}=\left.A_{p} \zeta\right|_{z=0^{+}} \\
& \left.\zeta\right|_{z=0^{-}}-\left.\zeta\right|_{z=0^{+}}=2\left(\left.u_{3}\right|_{z=0^{+}}-\left.u_{3}\right|_{z=0^{-}}\right) \\
& +2 \operatorname{Pr}\left(\left.\frac{\partial u_{3}}{\partial z}\right|_{z=0^{-}}-\left.\mu \frac{\partial u_{3}}{\partial z}\right|_{z-0^{+}}\right) \\
& -\gamma\left(\frac{\partial^{2} \phi_{s}}{\partial x^{2}}+\frac{\partial^{2} \phi_{s}}{\partial y^{2}}\right) \\
& \mu P r_{l}\left(\left.\frac{\partial u_{1}}{\partial z}\right|_{z=0^{+}}+\left.\frac{\partial u_{3}}{\partial x}\right|_{z=0^{+}}\right) \\
& -\operatorname{Pr}\left(\left.\frac{\partial u_{1}}{\partial z}\right|_{z=0^{-}}+\left.\frac{\partial u_{3}}{\partial x}\right|_{z=0^{-}}\right)=0 \\
& \mu \operatorname{Pr}_{l}\left(\left.\frac{\partial u_{2}}{\partial z}\right|_{z=0^{+}}+\left.\frac{\partial u_{3}}{\partial y}\right|_{z=0^{+}}\right) \\
& -\operatorname{Pr}_{l}\left(\left.\frac{\partial u_{2}}{\partial z}\right|_{z=0^{-}}+\left.\frac{\partial u_{3}}{\partial y}\right|_{z=0^{-}}\right)=0
\end{aligned}
$$

where equations 8 and 10 have been used to simplify equations 11-13.

Nontrivial harmonic solutions for $\phi_{s}, \mathbf{u}$, and $\zeta$, proportional to $e^{i \omega t}+i k_{1} x+i k_{2} y$, that satisfy equations 1 and 2 and the boundary and boundedness conditions at $z= \pm \infty$ are given by

$$
\begin{aligned}
\phi_{s} & =e^{i \omega x+i k_{1} x+i k_{2 \prime} \prime} \\
\zeta & =e^{i\left(u t+i k_{1} x+i k_{2 y} y\right.} \begin{cases}b_{1} e^{k z}-F r^{-1}, & z<0 \\
b_{2} e^{-k z-\rho F r^{-1},} & z>0\end{cases}
\end{aligned}
$$




$$
\begin{aligned}
u_{1} & =e^{i(\omega)+i k_{1} x+i k_{2 y}} \\
& \begin{cases}b_{3} e^{\alpha z}-i k_{1}(i \omega+k)^{-1} b_{1} e^{k z}, & z<0 \\
b_{4} e^{r z}-i k_{1}(i \omega \rho-k)^{-1} b_{2} e^{-k z}, & z>0\end{cases} \\
u_{2}=e^{i \omega t+i k_{1} x+i k_{2 y}} & \\
& \cdot \begin{cases}b_{5} e^{e z}-i k_{2}(i \omega+k)^{-1} b_{1} e^{k z}, & z<0 \\
b_{6} e^{r z}-i k_{2}(i \omega \rho-k)^{-1} b_{2} e^{-k z}, & z>0\end{cases} \\
u_{3}=e^{i \omega t+i k_{1} x+i k_{2} y} & \cdot \begin{cases}\left.b_{7} e^{q z}-k(i \omega)+k\right)^{-1} b_{1} e^{k z}, & z<0 \\
b_{8} e^{r z}+k(i \omega \rho-k)^{-1} b_{2} e^{-k z}, & z>0\end{cases}
\end{aligned}
$$

where the foregoing solution has been normalized by setting the coefficient of the harmonic dependence of $\phi_{s}$ to unity. Here, the signs of $k_{1}$ and $k_{2}$ may be either positive or negative, and we have employed the definitions $k=\left(k_{1}^{2}+k_{2}^{2}\right)^{1 / 2}$, and $q$ and $r$ are defined as $2 \operatorname{Pr} q=1+[1+4 \operatorname{Pr}(i \omega+$ $\left.\left.\operatorname{Pr}_{l} k^{2}\right)\right]^{1 / 2}$ and $2 \mu \operatorname{Pr}_{l}=1-\left[1+4 \mu \operatorname{Pr}_{l}(i \omega \rho+\right.$ $\left.\left.\mu \operatorname{Pr}_{l} k^{2}\right)\right]^{1 / 2}$.

Substituting this solution into the interface conditions $9-13$ and using equation 7 for $z \leqslant 0$ yields nine conditions for the eight coefficients $b_{1}-b_{8}$ and the complex frequency (dispersion relation) $i \omega(k)$. In particular, these conditions are given by

$$
\begin{aligned}
& i k_{1} b_{3}+i k_{2} b_{5}+q b_{7} \\
& =i k_{1} b_{4}+i k_{2} b_{6}+r b_{8}=0 \\
& b_{3}-i k_{1}(i \omega+k)^{-1} b_{1}-b_{4} \\
& +i k_{1}(i \omega \rho-k)^{-1} b_{2}=\left(\rho^{-1}-1\right) i k_{1} \\
& b_{5}-i k_{2}(i \omega+k)^{-1} b_{1}-b_{6} \\
& +i k_{2}(i \omega \rho-k)^{-1} b_{2}=\left(\rho^{-1}-1\right) i k_{2} \\
& b_{7}-k(i \omega+k)^{-1} b_{1}-\rho b_{8} \\
& \text { - } \rho k(i \omega \rho-k)^{-1} b_{2}=(1-\rho) i \omega \\
& b_{\bar{\tau}}-k(i \omega+k)^{-1} b_{1}-A_{p} b_{2} \\
& =i \omega-\rho \mathrm{Fr}^{-1} \mathrm{~A}_{p} \\
& {\left[1+k(i \omega+k)^{-1}\left(2 k P r_{1}-1\right)\right] b_{\downarrow}} \\
& -\left[1+k(i \omega \rho-k)^{-1}\left(2 k \mu \operatorname{Pr}_{l}+2-\rho\right)\right] b_{2} \\
& +\left(1-2 \operatorname{Pr}_{1} q\right) b_{7}-\left(2-\rho-2 \mu \operatorname{Pr}_{1}\right) b_{8} \\
& =(1-\rho)\left(F r^{-1}-i \omega\right)+\gamma k^{2} \\
& \left(\mu P r_{r} r-1\right) b_{4}+\left(2 k \mu P r_{1}+1\right)(i \omega \rho-k)^{-1} i k_{1} b_{2} \\
& +i k_{1} \mu \operatorname{Pr}_{1} b_{s}+\left(1-\operatorname{Pr}_{1} q\right) b_{3}+(2 \operatorname{Pr} k-1) \\
& (i \omega+k)^{-1} i k_{1} b_{1}-i k_{1} \operatorname{Pr} b_{7}=\left(\rho^{-1}-1\right) i k_{1}
\end{aligned}
$$

$$
\begin{aligned}
& \left(\mu \operatorname{Pr}_{l}-1\right) b_{6}+ \\
& \left(2 k \mu \operatorname{Pr}_{l}+1\right)(i \omega \rho-k)^{-1} i k_{2} b_{2} \\
& +i k_{2} \mu \operatorname{Pr}_{r_{8}}+\left(1-\operatorname{Pr}_{1}\right) b_{5} \\
& \quad+\left(2 \operatorname{Pr}_{l} k-1\right)(i \omega+k)^{-1} i k_{2} b_{1} \\
& \quad-i k_{2} \operatorname{Pr}_{2} b_{7}=\left(\rho^{-1}-1\right) i k_{2}
\end{aligned}
$$

Although the foregoing problem is linear in the coefficients $b_{1}-b_{8}$, explicit expressions for the dispersion relation $i \omega(k)$ and the neutral stability boundaries are not readily obtainable in closed form, except in certain special cases as noted later. However, it is possible to develop tractable perturbation expansions for these quantities in the realistic limit that the density and viscosity ratios $\rho$ and $\mu$ are small, as is $\mathrm{Fr}^{-1}$ in the case of reduced gravity.

\section{Asymptotic Analysis of the Cellular Stability Boundary}

It turns out that the $\rho \ll 1$ limit implies the existence of several different wave-number regimes [6], which in turn implies several different expansions for the dispersion relation. This is motivated by considering the solution of equations 18-25 in the limit of zero viscosity $\left(\mu=P r_{l}=0\right)$, which leads to a tractable form of the dispersion relation for arbitrary $\rho$. In particular, the neutral stability boundaries with respect to infinitesimal hydrodynamic disturbances proportional to $e^{i \omega t \pm t k \cdot x}$, where $\mathbf{k}$ and $\mathbf{x}$ are the transverse wave number and coordinate vectors, respectively, are given for $A_{p}<0$ by [5]

$$
\begin{aligned}
& A_{p}= \\
& \rho \frac{\rho(1-\rho) F r^{-1}+\rho \gamma k^{2}-(1-\rho) k}{\rho^{2}(3-\rho) F r^{-1}+\rho^{2} \gamma k^{2}+(1-\rho)(2-\rho) k} \\
& \quad \leq 0, \quad \omega=0
\end{aligned}
$$

and

$$
\begin{aligned}
& A_{p}=-\rho /(1-\rho), \quad \omega^{2}=k[(1+\rho) \\
& \left.\cdot(1-\rho)^{-1} F r^{-1}+k^{2}(1-\rho)^{-1} \gamma+k / \rho\right]
\end{aligned}
$$

where $k=|\mathbf{k}|$. For $A_{p}>0$, the basic solution is always unstable, and thus steady, planar combustion is only stable in the region $A_{p}<0$ that lies between these two curves (Fig. 1). The first of these boundaries is a cellular boundary $(\omega=0)$ that corresponds to the classical Landau instability. Indeed, in the limit $A_{p}=0$, corresponding to a constant normal burning rate independent of pressure, Landau's classical result is recovered since in the limit that $\gamma \mathrm{Fr}^{-1}$ approaches the value $(1-\rho) / 4 \rho^{2}$ from below, the 


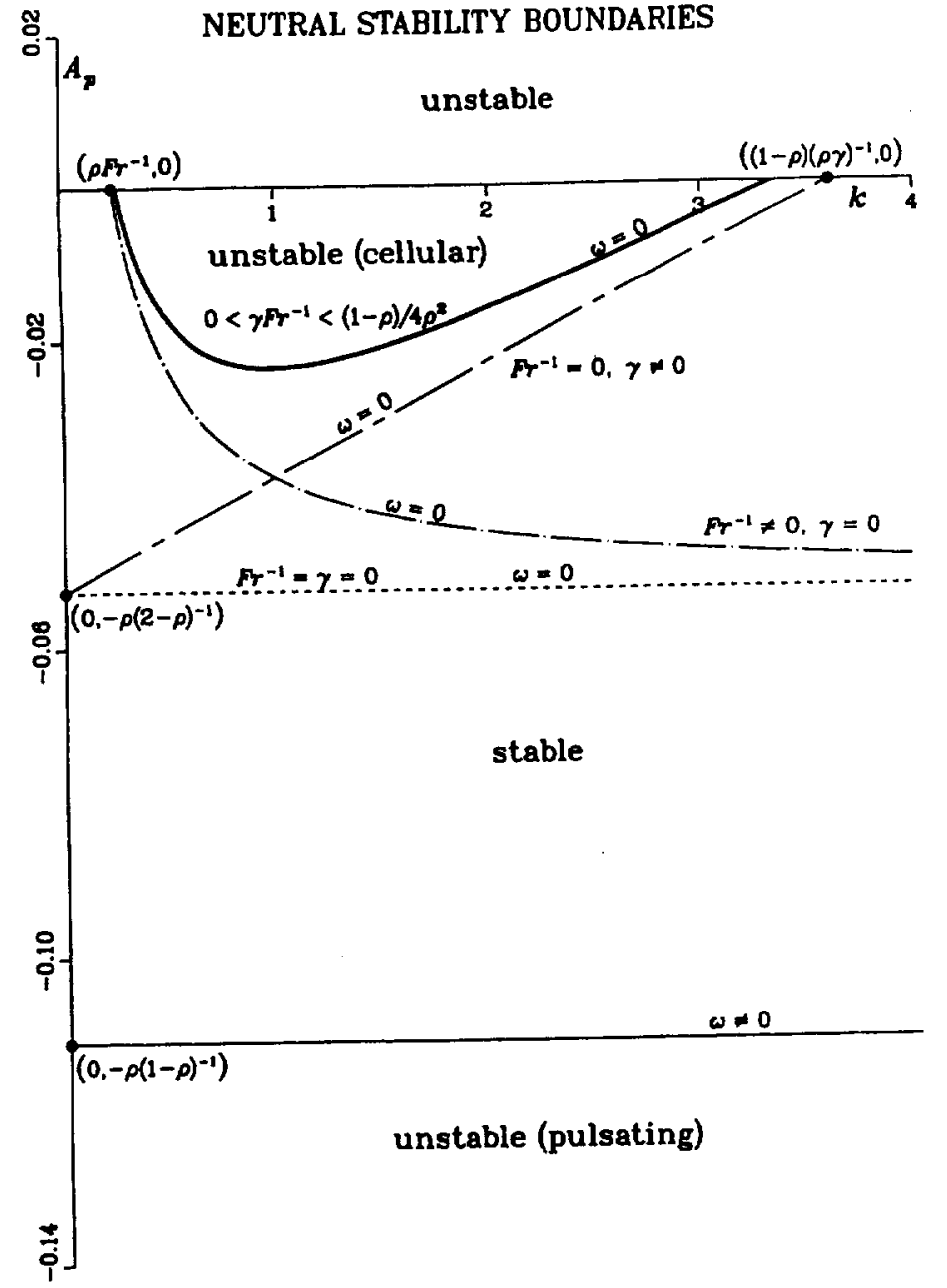

FIG. 1. Hydrodynamic neutral stability boundaries in the limit of zero viscosity. cellular stability boundary recedes from the region $A_{p}<0$. The pulsating stability boundary (27), on the other hand, only occurs for nonzero $A_{p}$ and, hence, was not predicted by the classical theories that assumed a constant normal burning rate. We note that zero and negative values of $A_{p}$ over certain pressure ranges are characteristic of the HAN-based liquid propellants mentioned earlier [2].

The formalism necessary to analyze the fully viscous problem is suggested by the fact that, for small $\rho$, the cellular boundary (26) has different limiting forms dependent on the relative magnitude of $k$ with respect to $\rho$. Thus, based on characteristic parameter values, we introduce a bookkeeping parameter $\varepsilon \ll$ 1 and define the scaled parameters $g^{*}, \rho^{*}, \mu^{*}$, and $A_{p}^{*}$ according to $\rho=\rho^{*} \varepsilon, \mu=\mu^{*} \varepsilon, A_{p}=A_{p}^{*} \varepsilon$, and either $F r^{-1}=g$ or $F r^{-1}=g^{*} \varepsilon$, where $\gamma$ and $P r_{l} \equiv$ $P$ are regarded as $\mathrm{O}(1)$ and the scaling for $A_{p}$ is motivated by equation 26 . Here, the second scaling for
$\mathrm{Fr}^{-1}$ corresponds to a reduced gravity limit, whereas the first definition indicates the normal gravity case. Equation 26 then suggests three wave-number scales; an inner (small) scale $k_{i}$, the outer $\mathrm{O}(1)$ scale $k$, and a far outer (large) scale $k_{f}=k \varepsilon$, where the inner scale is defined as $k_{i}=k / \varepsilon$ for $F^{-1} \sim \mathrm{O}(1)$ and $k_{i}=k / \varepsilon^{2}$ for the reduced gravity limit $F r^{-1} \sim$ $O(\varepsilon)$. Thus, in each of these regions, the corresponding leading-order expressions for $A_{p}^{*}$ are deduced from equation 26 as

$$
\begin{aligned}
& A_{\nu}^{*(i)} \sim\left\{\begin{array}{l}
\rho^{*}\left(\rho^{*} g-k_{i}\right) / 2 k_{i} \\
\rho^{*}\left(\rho^{*} g^{*}-k_{i}\right) / 2 k_{i}
\end{array}\right. \\
& A_{p}^{*(u)}--\frac{1}{2} \rho^{*}, \quad A_{\nu}^{*(f)}-\frac{1}{2} \rho^{*}\left(\rho^{*} \gamma k_{f}-1\right)
\end{aligned}
$$

and hence a uniformly valid composite expansion $A_{p}^{*}(c)(k)$ may be constructed as 


$$
\begin{aligned}
A_{p}^{*(c)} & \sim A_{p}^{*(i)}+A_{p}^{*(o)}+A_{p}^{*(f)} \\
& -\lim _{k_{i} \rightarrow \infty} A_{p}^{*(i)}-\lim _{k_{j} \rightarrow(l)} A_{p}^{*(f)} \\
& \sim-\frac{1}{2} \rho^{*}+\frac{1}{2} \varepsilon \rho^{* 2} \gamma k+\left\{\begin{array}{l}
\varepsilon \rho^{* 2} g / 2 k \\
\varepsilon^{2} \rho^{* 2} g^{*} / 2 k
\end{array}\right.
\end{aligned}
$$

where the definitions of $k_{i}$ and $k_{f}$ have been used to express the final result in terms of $k$, and the upper and lower expressions for $A_{p}^{*\left({ }^{i}\right)}$ correspond respectively to the normal and reduced gravity limits described earlier. In terms of the original unscaled parameters, equation 29 becomes $2 A_{p} \sim-\rho+\gamma p^{2} k$ $+F r^{-1} \rho^{2} / k$, which, in the parameter regime considered, is a leading-order asymptotic representation of the exact relation 26 . It is readily deduced from equations 28 and 29 , as discussed in further detail elsewhere [6], that surface tension stabilizes large wave-number disturbances, while gravity stabilizes small wave-number perturbations. In the reducedgravity limit, the minimum in the stability boundary is thus shifted to smaller wave numbers, and thus the hydrodynamic cellular instability becomes a long-wave instability phenomenon (Fig. 2).

Corresponding results may be obtained for the vis cous case. However, to deduce the asymptotic forms of both the cellular and the pulsating stability boundaries, it is preferable from the standpoint of tractability to introduce the scalings introduced previously and appropriate perturbation expansions for the coefficients $b_{i}$ directly into equations $18-25$ for each wave-number regime, and to obtain the neutral stability boundary in question from the expanded form of those equations. Thus, for the cellular boundary $A_{p}^{*}(k)[6]$, we deduce the following expansions and results for each wave-number regime.

$$
\begin{gathered}
\text { (i) } k \sim O(1) \\
r \sim r_{1} \varepsilon+\cdots, \quad r_{1}=\mu^{*} P k^{2} \\
q \sim q_{0}+\cdots \\
q_{0}=(2 P)^{-1}\left[1+\left(1+4 P^{2} k^{2) 1 / 2}\right]\right. \\
b_{i} \sim b_{i}^{(-1)} \varepsilon^{-1}+b_{i}^{(0)}+b_{i}^{(1)} \varepsilon+\cdots \\
\left.i=2,8 ; b_{i} \sim b_{i}^{(0)}+b\right\}^{(1)} \varepsilon+\cdots \\
i=1,3,4,5,6,7 \\
A_{p}=A_{p}^{*(o)} \varepsilon \sim \varepsilon\left(A_{0}^{*(o)}+A_{i}^{*(o)} \varepsilon+\cdots\right) \\
A_{0}^{*(o)}=-\rho^{* / 2} \\
\text { (ii) } k=\left\{\begin{array}{l}
k_{i} \varepsilon \\
k_{i} \varepsilon^{2}
\end{array}\right.
\end{gathered}
$$

$$
\begin{aligned}
& r \sim \mu^{*} P k_{i}^{2}\left\{\begin{array}{l}
\varepsilon^{3}+\cdots \\
\varepsilon^{5}+\cdots
\end{array}\right. \\
& q-(2 P)^{-1}+P k_{i}^{2}\left\{\begin{array}{l}
\varepsilon^{2}+\cdots \\
\varepsilon^{4}+\cdots
\end{array}\right. \\
& b_{i} \sim\left\{\begin{array}{l}
b_{i}^{(0)}+b_{i}^{(1)} \varepsilon+\cdots \\
b_{i}^{(1)} \varepsilon+b_{i}^{(2)} \varepsilon^{2}+\cdots, i=2,8
\end{array}\right. \\
& b_{i} \sim\left\{\begin{array}{l}
b_{i}^{(1)} \varepsilon+b_{i}^{(2)} \varepsilon^{2}+\cdots \\
b_{i}^{(2)} \varepsilon^{2}+b_{i}^{(3)} \varepsilon^{3}+\cdots
\end{array}\right. \\
& i=1,3,4,5,6,7 \\
& A_{p}=A_{p}^{*(i)} \varepsilon \sim \varepsilon\left(A_{0}^{*(i)}+A_{1}^{*(i)} \varepsilon+\cdots\right) \\
& A_{0}^{*(i)} \sim\left\{\begin{array}{l}
\rho^{*}\left(\rho^{*} g-k_{i}\right) / 2 k_{i} \\
\rho^{*}\left(\rho^{*} g^{*}-k_{i}\right) / 2 k_{i}
\end{array}\right. \\
& \text { (iii) } k=k_{\mathrm{f}} / \varepsilon \\
& r \sim r_{(-1)} \varepsilon^{-1}+\cdots \\
& r_{(-1)}=\frac{1-\left(1+4 \mu^{* 2} p^{2} k_{j}^{2}\right)^{1 / 2}}{2 \mu^{*} P} \\
& q \sim q_{(-1)} \varepsilon^{-1}+\cdots, \quad q_{(-1)}=k_{f} \\
& b_{i} \sim b_{i}^{(-1)} \varepsilon^{-1}+b_{i}^{(0)}+\cdots, \quad i=1,3,5,7 \\
& b_{i} \sim b_{i}^{(-2)} \varepsilon^{-2}+b_{i}^{(-1)} \varepsilon^{-1}+\cdots \\
& i=2,4,6,8 \\
& A_{p}=A_{p}^{*(f)} \varepsilon-\varepsilon\left(A_{0}^{*}(f)+A_{1}^{*(f)} \varepsilon+\cdots\right)_{;} A_{0}^{*}(f)--\rho^{*} \\
& +\frac{2 \rho^{*} \mu^{*} P\left[1+k_{f}\left(\rho^{*} \gamma+2 \mu^{*} P+2 \rho^{*} P\right)\right]}{4 \mu^{*} P\left(1+\rho^{*} P k_{f}\right)-\left[1-\left(1+4 \mu^{* 2} P^{2} k_{f}^{2}\right)^{1 / 2}\right]\left(\rho^{*} \gamma+2 \mu^{*} P\right)}
\end{aligned}
$$

We observe that the leading-order results 32 and 35 are equivalent to the corresponding inviscid results 28 . Thus, to leading order, neither the inner nor the outer wave-number regimes are influenced by viscous effects, which, to a first approximation, are only significant for large wave-number disturbances. This is reflected in the leading-order expression for the cellular stability boundary given by equation 38 , where, among other features, it is readily observed that both the liquid and the gas-phase viscosities (through the parameters $P$ and $\mu^{*} P$, respectively) enter into this expression, reflecting an equal influence of viscous and surface-tension effects on the neutral stability boundary in the large wave-number regime. The equal importance of gasphase viscosity relative to that of the liquid phase stems from the fact that gas-phase disturbances are, according to equations 37 , larger in magnitude than those in the liquid phase, such that a weak damping of a larger magnitude disturbance is as significant as 


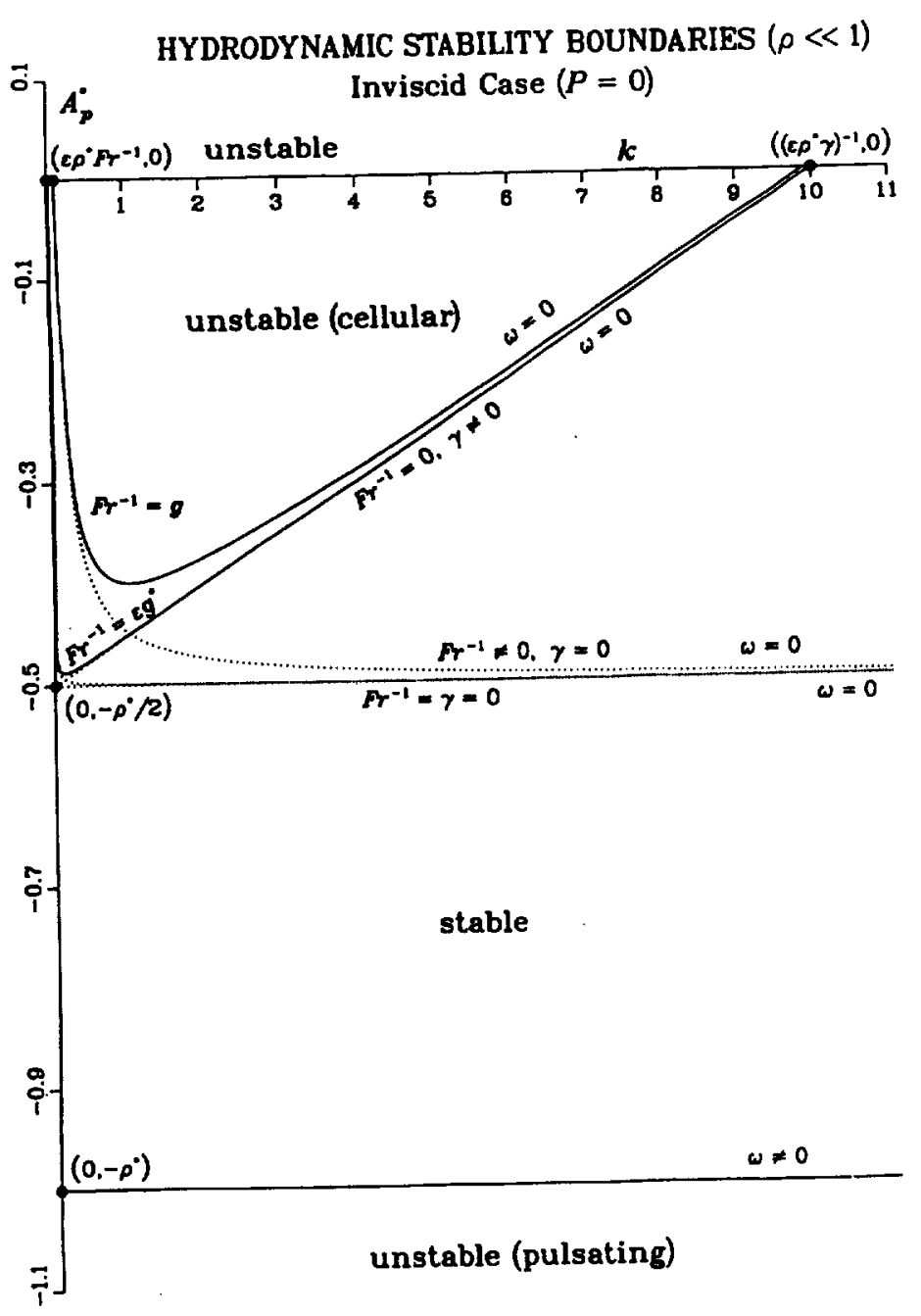

FIG. 2. Asymptotic representation of the cellular hydrodynamic neutral stability boundary in the limit of zero viscosity. The upper (lower) solid curves correspond to the two cases described by equations 29 for normal and reduced-gravity regimes, respectively (curves drawn for the case $\varepsilon=0.04, \rho^{*}=1.0, \mathrm{~g}=2.5$, $\mathrm{g}^{*}=1.0$ ). an $O(1)$ damping of a smaller magnitude disturbance. In the limit $P \rightarrow 0$, the inviscid expression 28 is recovered. It is easily shown that $\lim _{k \rightarrow 0} A_{0}^{*}(f)=$ $-\rho^{*} / 2$, so that the far outer solution can be matched to the outer solution 32 . Indeed, a uniformly valid composite expansion spanning all three wave-number regimes may be constructed as in the inviscid case, giving the result

$$
\begin{aligned}
& A_{\mu}^{*(c)}--\rho^{*}+ \\
& \frac{2 \rho^{*} \mu^{*} P\left[1+\varepsilon k\left(\rho^{*} \gamma+2 \mu^{*} P+2 \rho^{*} P\right)\right]}{4 \mu^{*} P\left(1+\varepsilon k \rho^{*} P\right)-\left(\rho^{*} \gamma+2 \mu^{*} P\right)\left[1-\left(1+4 \mu^{* 2} P^{2} \varepsilon^{2} k^{2}\right)^{1 / 2}\right]} \\
& +\frac{\rho^{* 2}}{2 k}\left\{\begin{array}{l}
\varepsilon g \\
\varepsilon^{2} g^{*}
\end{array}\right.
\end{aligned}
$$

or, reverting to unscaled parameters,

$$
\begin{aligned}
& A_{p}^{(c)}--\rho+ \\
& \frac{2 \rho \mu P[1+k(\rho \gamma+2 \mu P+2 \rho P)]}{4 \mu P(1+k \rho P)-(\rho \gamma+2 \mu P)\left[1-\left(1+4 \mu^{2} P^{2} k^{2}\right)^{1 / 2}\right]} \\
& +\frac{\rho^{2}}{2 k} F^{-1}
\end{aligned}
$$

The cellular stability boundaries, based on equation 39 , are reproduced in Fig. 3, where only those portions of the curves that lie in the region $A_{p}^{*} \leq 0$ are shown. For sufficiently small positive values of $A_{p}$, it may be shown that there always exists a positive (real) root $i \omega$ of the dispersion relation, which implies that this region is intrinsically unstable. We note from equation 38 that as $k_{f}$ increases, $A_{0}^{*(f)}$ increases, intersecting the $A_{0}^{*}(f)=0$ axis at the value $k_{f}=\left(\rho^{*} \gamma\right)^{-1}\left[1-\mu^{*} P /\left(\rho^{*} \gamma+2 \mu^{*} P\right)\right]$, which agrees 


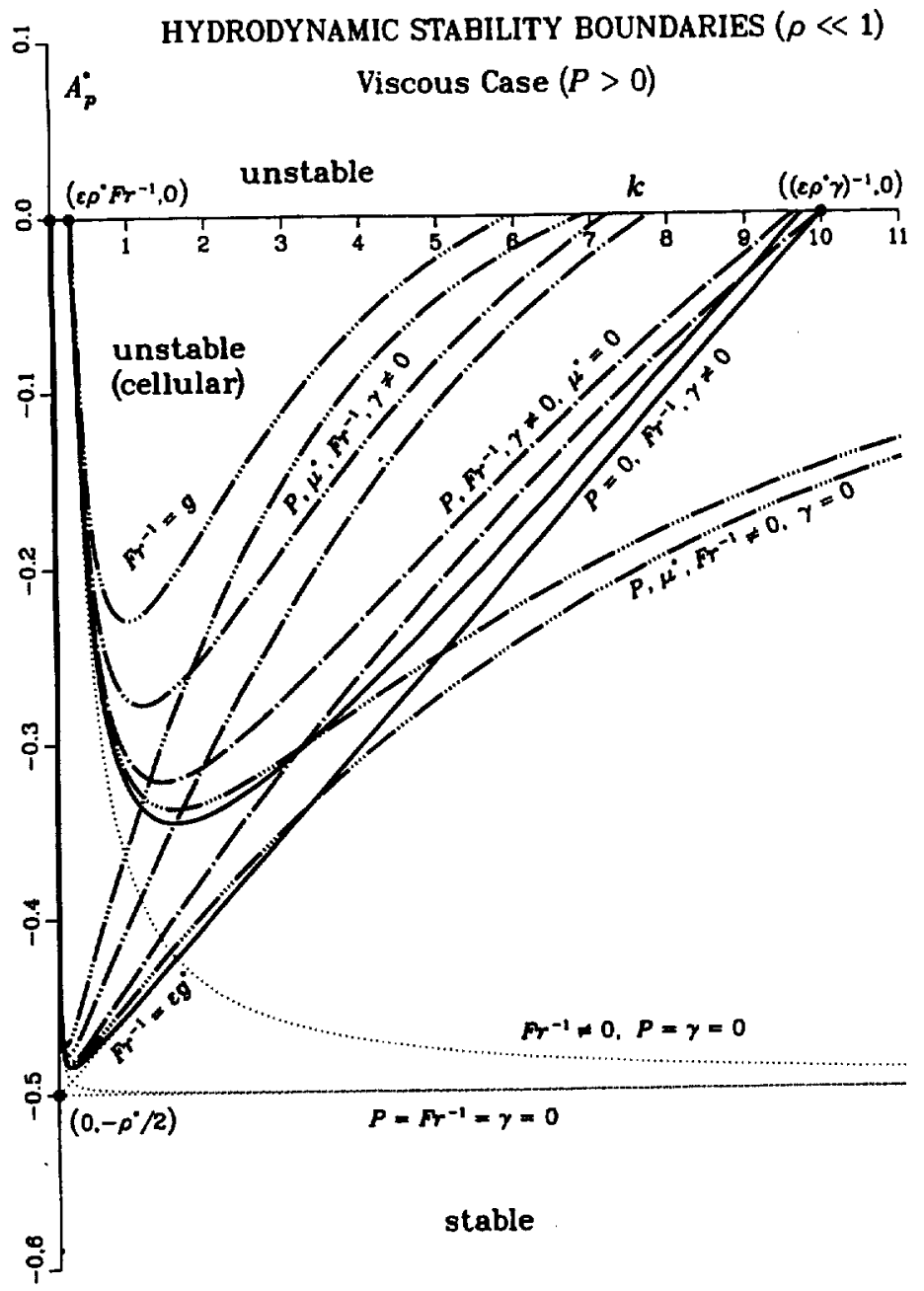

Fic. 3. Asymptotic representation of the cellular hydrodynamic neutral stability boundary for the viscous case. The upper and lower sets of curves correspond to the normal and reduced-gravity regimes, respectively, in the asymptotic limit considered in this work (curves drawn for the case $\varepsilon=.04, \rho^{*}=1.0, g=6.0$. $\left.g^{*}=2.0\right\rangle$. The solid curves correspond to the inviscid limit $(P=0)$ with nonzero surface tension $(\gamma=$ 2.5). The dash-dot curves correspond to nonzero surface tension $(\gamma$ $=2.5)$ and liquid viscosity $(P=1.0)$ but zero gas-phase viscosity $\left(\mu^{*} P=\right.$ $0)$. The dash-dot-dot curves differ from the dash-dot curves by the addition of gas-phase viscosity $\left(\mu^{*} P=\right.$ 1.0) and are similar to the dash-dotdot-dot curves, where the latter correspond to larger viscosities $(\boldsymbol{P}=$ $\mu^{*} P=2.0$ ). The dash-dot-dot-dotdot curves correspond to a viscous case $\left(P=\mu^{*} P=1.0\right)$ but with zero surface tension $(\gamma=0)$, so that, from equation 38 , the curves do not intercept the $A_{p}^{*}=0$ axis. with the inviscid result in the limit $\mu^{*} P \rightarrow 0$. It is readily seen from Fig. 3 that, as in the inviscid case, the essential qualitative difference between the normal and reduced-gravity curves is the location of the critical wave number for instability. Specifically, the minimum in the neutral stability boundaries occurs for $O(1)$ values of $k$ under normal gravity, and at $k \sim O\left(\varepsilon^{1 / 2}\right)$ in the reduced-gravity limit considered here. It is also clear from Fig. 3 that increasing the values of any of the parameters $P, \mu^{*} P$, or $\gamma$ serves to shrink the size of the unstable domain through damping of short-wave perturbations. The non-negligible effects of gas-phase viscosity represents an important correction to Levich's original treatment [3] in which these effects were simply assumed to be small. The results 39-40 thus synthesize and significantly extend the classical Landau-Levich results $[1,3]$, not only in allowing for a dynamic dependence of the burning rate on local conditions in the vicinity of the liquid-gas interface but also in its formal treatment of those processes (surface tension, liquid and gas-phase viscosity) that affect damping of large wave-number disturbances.

\section{Asymptotic Analysis of the Pulsating Stability Boundary}

As indicated previously, the existence of a nonstationary pressure dependence on the burning rate (i.e., $A_{p} \neq 0$ leads to the prediction of a pulsating hydrodynamic stability boundary that is absent when such a pressure coupling is neglected, as in the original Landau-Levich theories. In the inviscid case, this boundary (equation 27) is a straight line that lies below the cellular boundary discussed earlier, but this is modified under the influence of viscosity, as we shall demonstrate.

For the scalings adopted in the preceding section 
[in particular, for $P \sim O(1), \mu \sim O(\varepsilon)$ ], it tums out that, unlike the cellular stability boundary for which viscous effects only have a leading-order effect in the far outer wave-number regime, the effects of viscosity have a leading-order effect on the pulsating boundary for $O(1)$ wave numbers as well. Thus, in the outer wave-number region, we seek a solution for the dispersion relation in the form $i \omega \sim \varepsilon^{-1 / 2}\left(i \omega_{0}\right.$ $\left.+i \omega_{1} \varepsilon^{1 / 4}+i \omega_{2} \varepsilon^{1 / 2}+\cdots\right)$, where the leading-order term is suggested by the explicit results for the inviscid case $[5,6]$, and the expansion in powers of $\varepsilon^{1 / 4}$ is suggested by the expansions for $r$ and $q$ given below equation 17 , which have the form

$$
\begin{gathered}
r \sim r_{(1 / 2)} \varepsilon^{1 / 2}+r_{(3 / 4)} \varepsilon^{3 / 4}+r_{1} \varepsilon+\cdots \\
r_{(1 / 2)}=-i \omega_{0} \rho^{*}, \quad r_{(3 / 4)}=-i \omega_{1} \rho^{*} \\
r_{1}=-i \omega_{2} \rho^{*}-\left(\mu^{*} P k\right)^{2} \\
q-q_{(-1 / 4)} \varepsilon^{-1 / 4}+q_{0} \varepsilon^{1}+\cdots \\
q_{(-1 / 4)}=\left(i \omega_{0} / P\right)^{1 / 2} \\
q_{0}=(2 P)^{-1}\left[1+i \omega_{1} /\left(i \omega_{0} P\right)^{1 / 2}\right]
\end{gathered}
$$

Corresponding expansions for the coefficients $b_{i}$ in equations 18-25 are determined as

$$
\begin{aligned}
& b_{i}=b_{i}^{(-1)} \varepsilon^{-1}+b_{i}^{(-3 / 4)} \varepsilon^{-3 / 4} \\
& +b_{i}^{(-1 / 2)} \varepsilon^{-1 / 2}+\cdots, \quad i=1,2,8 \\
& b_{1}=b_{i}^{(-1 / 2)} \varepsilon^{-1 / 2}+b_{i}^{(-1 / 4)} \varepsilon^{-1 / 4}+\cdots \\
& i=3,4,5,6 \text {; } \\
& b_{i}=b_{i}^{(-1 / 4)} \varepsilon^{-1 / 4}+b_{i}^{(0)} \varepsilon^{0}+\cdots, i=7
\end{aligned}
$$

where the leading terms in the expansions for $b_{1}, b_{2}$ $b_{4}, b_{6}$, and $b_{8}$ are consistent with the inviscid results $[5]$ and the remaining coefficients appear only for nonzero values of $P$ and are conservatively postulated to have the indicated expansions. Substituting these expansions into equations 18-25 and equating coefficients of like powers of $\varepsilon$, we obtain the leading-order equations and results

$$
\begin{gathered}
i k_{1} b_{3}^{(-1 / 2)}+i k_{2} b_{5}^{(-1 / 2)}+q_{(-1 / 4)} b_{7}^{(-1 / 4)}=0 \\
=i k_{1} b_{4}^{(-1 / 2)}+i k_{2} b_{6}^{(-1 / 2)}+r_{(1 / 2)} b_{8}^{-1} \\
b_{1}^{(-1)}+b_{2}^{(-1)}-2 b_{8}^{(-1)}=0 \\
b_{2}^{(-1)}=-k / \rho^{*}, \quad b_{1}^{(-1)}=\left(i \omega_{0}\right)^{2} / k \\
b_{8}^{(-1)}=-\left(k / \rho^{*}\right)\left(1+A_{p}^{*} / \rho^{*}\right)
\end{gathered}
$$

where the last of equations 46 was obtained from the leading-order difference of equations 21 and 22 , and the remainder of the leading-order versions of equations $18-25$ give redundant results. Combining equations 46 , we obtain

$$
\left(i \omega_{10}\right)^{2}=\left(k^{2} / \rho^{*}\right)\left(1+2 A_{p}^{*} / \rho^{*}\right)
$$

and thus $\left(i \omega_{0}\right)^{2} \gtrless 0$ for $A_{p}^{*} \gtrless-\rho^{*} / 2$, which essentially recovers the leading-order cellular stability boundary (32) for $\mathrm{O}(1)$ wave numbers, but gives no information on the pulsating boundary because $i \omega_{0}$ is purely imaginary for $A_{p}^{*}<-\rho^{*} / 2$. Hence, stability in the latter region is determined by higher-order coefficients in the foregoing expansion for $i \omega$.

Continuing with the analysis of the expanded forms of equations 18-25, we obtain the second-order equations and results

$$
\begin{gathered}
i k_{1} b_{3}^{(-1 / 4)}+i k_{2} b_{5}^{(-1 / 4)}+q_{(-1 / 4)} b_{7}^{(0)} \\
+q_{0} b_{7}^{(-1 / 4)}=0 \\
i k_{1} b_{4}^{(-1 / 4)}+i k_{2} b_{6}^{(-1 / 4)}+r_{(1 / 2)} b_{8}^{(-3 / 4)} \\
+r_{(3 / 4)} b_{8}^{(-1)}=0 \\
k b_{2}^{(-1 / 2)}-q_{(-1 / 4)} b_{7}^{(-1 / 4)}=i \omega_{0} k\left(1-A_{p}^{*} / \rho^{*}\right) \\
b_{7}^{(-1 / 4)}-\left(k / i \omega_{0}\right) b_{1}^{(-3 / 4)}=i \omega_{1} \\
b_{1}^{(-3 / 4)}=b_{2}^{(-3 / 4)}=b_{8}^{(-3 / 4)=b_{3}^{(-1 / 2)}} \\
=b_{5}^{(-1 / 2)}=0
\end{gathered}
$$

where the first of equations 50 was obtained from the sum of equation 19 multiplied by $i k_{1}$ and equation 20 multiplied by $i k_{2}$ and the first of equations 45 , the fact that $b_{8}^{(-3 / 4)}=0$ follows from the difference of equations 21 and 22 , and the last two of equations 51 follow from equations 24 and 25 in conjunction with the result $b_{2}^{(-3 / 4)}=0$. From these results and the first of equations 45 , we thus conclude that

$$
\begin{aligned}
& b_{t}^{(-1 / 4)}=i \omega_{1}=0, \\
& b_{2}^{(-1 / 2)}=i \omega_{0}\left(1-A_{p}^{*} / \rho^{*}\right)
\end{aligned}
$$

where the fact that $i \omega_{1}=0$ implies the need to calculate $i \omega_{2}$ to determine stability in the region $A_{p}^{*}<-\rho^{*} / 2$. Proceeding in this fashion, we obtain from the next-order versions of equations 21 and 23 the difference of equations 21 and 22 , and the sum of equation 24 multiplied by $i k_{1}$ and equation 25 multiplied by $i k_{2}$, the relations

$$
\begin{aligned}
& b_{7}^{(0)}-\left(k / i \omega_{0}\right) b_{1}^{(-1 / 2)}+ \\
& {\left[\left(i \omega_{2}+k\right) /\left(i \omega_{0}\right)^{2}\right] k b_{1}^{(-1)}} \\
& \quad-\rho^{*} b_{8}^{(-1)}+\rho^{*} b_{2}^{(-1)}=i \omega_{2} \\
& b_{1}^{(-1 / 2)}+\left(k / i \omega_{0}\right)(2 P k-1) b^{(-1)}+b_{2}^{(-1 / 2)} \\
& \quad+\left(2 i \omega_{0} \rho^{*} / k\right) b_{2}^{(-1)}-2 b_{8}^{(-1 / 2)}=-i \omega_{0}
\end{aligned}
$$




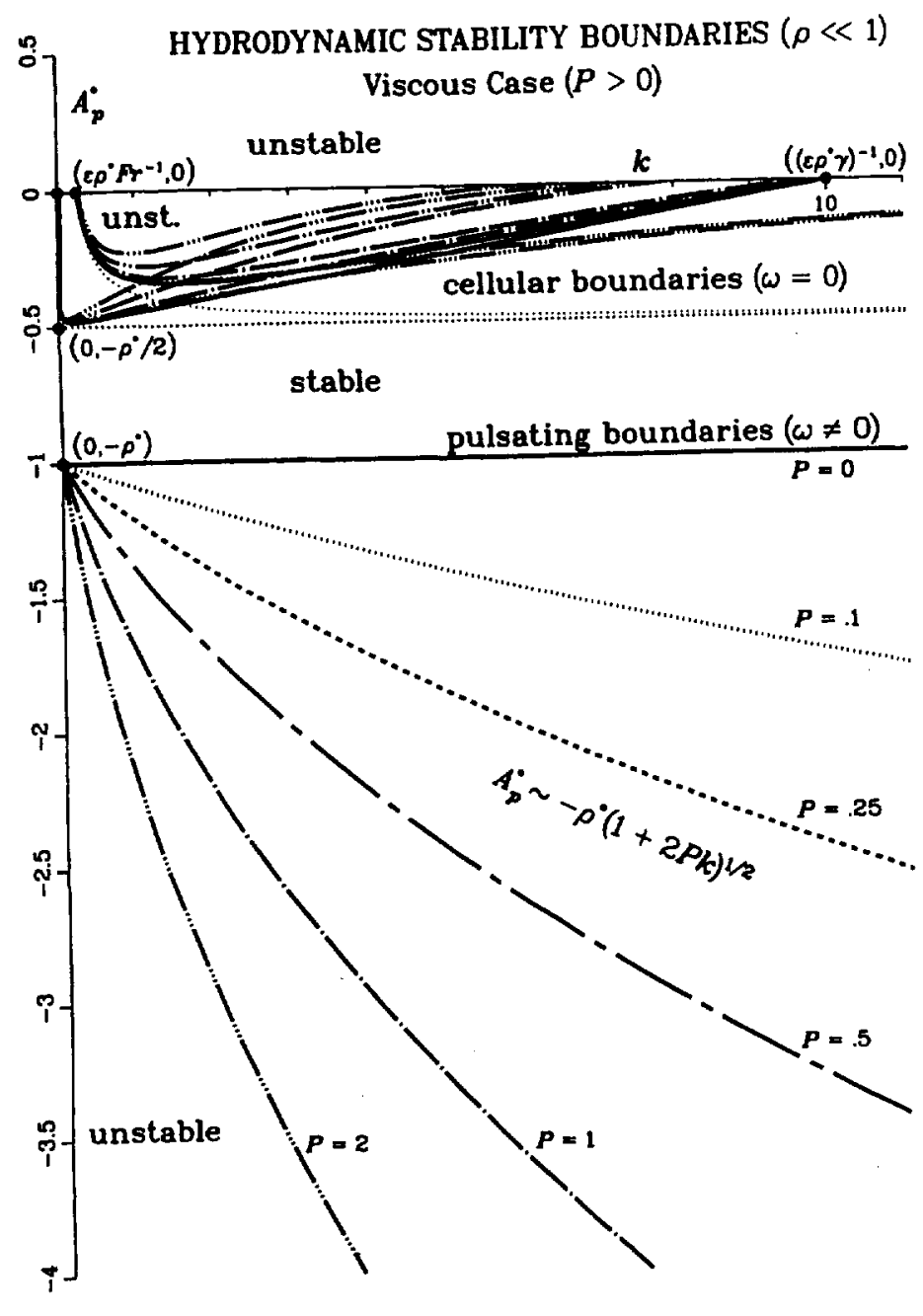

FIG. 4. Asymptotic representation of the pulsating hydrodynamic neutral stability boundary for the viscous case $(P>0)$. The region between the pulsating and cellular boundaries (the latter are shown on an expanded scale in Fig. 3) is the stable region with respect to hydrodynamic instability.

$$
\begin{aligned}
& -\rho^{*} b_{8}^{(-1 / 2)}+\rho^{*} b_{2}^{(-1 / 2)}+\left(i \omega_{0} \rho^{* 2 / k}\right) b_{2}^{(-1)} \\
& +A_{p}^{*} b_{2}^{(-1 / 2)}=-i \omega_{0} \rho^{*} \\
& -i \omega_{0} \rho^{*} b_{8}^{(-1)}+k b_{2}^{(-1 / 2)}+i \omega_{0} \rho^{*} b_{2}^{(-1)} \\
& +i \omega_{0} b_{7}^{(0)}-(2 P k-1)\left(k^{2} / i \omega_{0}\right) b_{1}^{(-1)}=0
\end{aligned}
$$

which, when combined with the expressions for $b_{1}^{(-1)}, b_{2}^{(-1)}, b_{\xi}^{(-1)}, b_{2}^{(-1 / 2)}$, and $i \omega_{0}$ given earlier, constitute four equations for the four unknowns $b_{1}^{(-1 / 2)}, b_{7}^{(0)}, b_{8}^{(-1 / 2)}$, and $i \omega_{2}$. Solving these simultaneous equations, we thus obtain

$$
\begin{aligned}
& b_{j}^{(0)}=-2 P k^{2} \text {. } \\
& b_{1}^{(-1 / 2)}=\left[2 P k+1+A_{p}^{*} / \rho^{*}-2\left(A_{p}^{*} / \rho^{*}\right)^{2}\right] i \omega_{0} \\
& b_{u}^{(-1 / 2)}=\left[1-\left(A_{p}^{*} / \rho^{*}\right)^{2}\right] i \omega_{0}
\end{aligned}
$$

$i \omega_{2}=k\left[\left(A_{p}^{*} / \rho^{*}\right)^{2}-2 P k-1\right]$

Equation 58 is the desired result, from which we conclude that $i \omega_{2} \lessgtr 0$ for $\left(A_{p}^{*} / \rho^{*}\right)^{2} \lessgtr 1+2 P k$. Thus, in the region $A_{p}^{*}<0, i \omega_{2}$ vanishes on the boundary

$$
A_{p}^{*} \sim-\rho^{*}(1+2 P k)^{1 / 2}
$$

which is a pulsating boundary (Fig. 4) because, from equation $47, i \omega_{0}$ is purely imaginary along this curve. Equation 59 is valid for $O(1)$ wave numbers, but because it matches to the leading-order inviscid inner pulsating boundary $A_{p}^{*} \sim-\rho^{*}$ as $k \rightarrow 0$ and becomes large in a negative sense as $k$ becomes large, it is clear that equation 59 represents the pulsating boundary for arbitrary wave numbers. That is, for $P \sim O(1)$, the effects of (liquid) viscosity on the pulsating boundary are, to a first approximation, absent for small wave numbers, are first felt for $O(1)$ 
wave-number perturbations, and are sufficient to move this boundary to larger-magnitude values $A_{p}^{*} \sim \mathrm{O}\left(\varepsilon^{-1 / 2}\right)$ in the far outer wave-number regime. In contrast, the cellular boundary 39 is unaffected for $O(1)$ and smaller wave numbers and is only modified an $\mathrm{O}(1)$ amount for $\mathrm{O}\left(\varepsilon^{-1}\right)$ wave numbers. Thus, the hydrodynamic pulsating boundary is more sensitive to viscous effects than is the corresponding cellular stability boundary. For smallermagnitude viscosities such that $P=\hat{P} \varepsilon \sim O(\varepsilon)$, it may be shown by an analogous calculation (Appendix) that $\mathrm{O}(1)$ modifications to the pulsating boundary then occur in the far outer wave-number regime according to $A_{p}^{*} \sim-\rho^{*}\left(1+2 \hat{P} k_{f}\right)^{1 / 2}$, which, in terms of unscaled quantities, is the same as equation 59.

\section{Conclusion}

The present work has presented a formal asymptotic treatment of hydrodynamic instability for a surface model of liquid-propellant combustion in which burning takes place at the liquid-gas interface. The model itself is based on a synthesized version of the classical models analyzed by Landau [1] and Levich [3], generalized to allow a coupling of the burning rate with the local pressure field [4,5]. The realistic smallness of the gas-to-liquid density ratio proved to be a convenient small parameter upon which to base an asymptotic treatment, resulting in three distinct wave-number regimes with different physical processes assuming dominance in each. Both cellular and pulsating hydrodynamic stability boundaries are predicted by the present model, the former corresponding to Landau's original notion of hydrodynamic instability and the latter representing a new prediction arising from the pressure dependence of the burning rate. For the cellular type of instability, it was shown that the gravitational acceleration (assumed to be normal to the undisturbed liquid-gas interface in the direction of the liquid) is responsible for stabilizing long-wave disturbances, whereas surface tension and viscosity are effective in stabilizing short-wave perturbations. In the case of pulsating instability, neither gravity nor surface tension play a leading-order role, and viscous effects are the dominant stabilizing influence. Indeed, for $O(1)$ liquid Prandtl numbers, the stabilizing effects of (liquid) viscosity on pulsating instability are significant for disturbances whose wave numbers are $O(1)$ and higher. On the other hand, viscous effects are only significant for large wave-number disturbances in the case of cellular instability, where the influence of gas and liquid viscosity are comparable despite the small ratio of these two parameters. Although the onset of pulsating hydrodynamic instability is predicted to occur only for sufficiently negative values of the pressure-sensitivity coefficient $A_{p}$, the persistence of the pulsating stability boundary (in the presence of viscous effects) for small wave numbers suggests that it should be observable in those types of liquid propellants, such as those based on hydroxylammonium nitrate (HAN) and triethanolammonium nitrate (TEAN), that are characterized by negative pressure sensitivities over certain pressure ranges. In connection with this, we note that sloshing behavior has been observed during combustion of certain HAN-TEAN-water mixtures [2], but because nonsteady burning can arise via secondary and higher-order bifurcations in the cellular region [8], as well as from a primary crossing of the pulsating boundary described here, further measurements are generally needed to determine the precise origin of such behavior in any given experiment.

\section{Nomenclature}

A burning rate

$A_{p} \quad$ pressure-sensitivity coefficient

$b_{i} \quad$ coefficients in perturbation solution $(i=$ $1,2, \ldots, 8)$

$\begin{array}{ll}\text { e } & \text { rate-of-strain tensor } \\ \text { Fr } & \text { Froude number }\end{array}$

Fr inverse Froude number (gravitational acceleration)

$k$ perturbation wave number

$\hat{\mathbf{n}}_{\mathrm{s}} \quad$ unit normal

$p \quad$ pressure

$P, \operatorname{Pr} \quad$ Prandtl number

$q \quad$ quantity defined below equation (17)

$r$ quantity defined below equation (17)

$t$ time variable

u perturbation velocity vector

$v$ velocity vector

$(x, y, z)$ moving coordinate system

$\gamma \quad$ surface-tension coefficient

$\varepsilon \quad$ small bookkeeping parameter

$\zeta$ perturbation pressure

$\lambda$ gas-to-liquid thermal diffusivity ratio

$\mu \quad$ gas-to-liquid viscosity ratio

$\rho \quad$ gas-to-liquid density ratio

$\phi_{s}$ perturbation in location of gas-liquid interface

$\Phi_{s} \quad$ location of gas-liquid interface

$\omega \quad$ complex perturbation frequency

\section{Subscripts, Superscripts:}

$i \quad$ inner wave-number regime or integer variable

$f \quad$ far outer wave-number regime liquid

gas outer wave-number regime scaled quantity 
Appendix. The Pulsating Stability Boundary for Small Liquid Prandlt Numbers

For small liquid viscosities such that $P=\hat{P} \varepsilon$ and the same scalings introduced in the $P \sim O(1)$ case analyzed in the main body of the text, the effect of viscosity on the cellular boundary disappears at leading order $(P \rightarrow 0$ in equations 39 and 40$)$, while the effect of viscosity on the pulsating boundary is only significant in the far outer wave-number regime. In that case, the appropriate expansions analogous to those introduced for the $P \sim \mathrm{O}(1)$ case are given by $i \omega \sim \varepsilon^{-3 / 2}\left(i \omega_{0}+i \omega_{1} \varepsilon^{1 / 4}+i \omega_{0} \varepsilon^{1 / 2}+\cdots\right)$ and

$$
\begin{aligned}
& r \sim r_{(-1 / 2)} \varepsilon^{1 / 2}+O\left(\varepsilon^{-1 / 4}\right), \quad r_{(-1 / 2)}=-i \omega_{1} \rho^{*} \\
& q \sim q_{(-5 / 4)} \varepsilon^{-5 / 4}+O\left(\varepsilon^{-1}\right), \quad q_{(-5 / 4)}=\left(i \omega_{1} / P\right)^{1 / 2} \\
& b_{i}=b_{i}^{(-2)} \varepsilon^{-2}+b_{i}^{(-\pi / 4)} \varepsilon^{-7 / 4} \\
& +b_{i}^{(-3 / 2)} \varepsilon^{-3 / 2}+\ldots, i=1,2,8 \\
& b_{l}=b_{i}^{(-3 / 2)} \varepsilon^{-3 / 2}+b_{i}^{(-5 / 4)} \varepsilon^{-5 / 4}+\ldots \\
& i=3,4,5,6 \\
& b_{i}=b_{i}^{(-5 / 4)} \varepsilon^{-5 / 4}+b_{i}^{(-1)} \varepsilon^{-1}+\ldots, i=7
\end{aligned}
$$

Substituting these expansions into equations $18-25$ and equating terms corresponding to like powers of $\varepsilon$ then gives, as previously, a sequence of equations for the recursive determination of the coefficients in the foregoing expansions. Similar to the calculation for $P \sim O(1)$ in the outer wave-number regime, we obtain in this case that

$$
\begin{aligned}
\left(i \omega_{0}\right)^{2} & =2\left(k_{f}^{2} / \rho^{* 2}\right)\left(A_{p}^{*}-\hat{A}_{p}^{*}\right), \quad i \omega_{1}=0, \\
i \omega_{2} & =k_{f}\left[\left(A_{p}^{*} / \rho^{*}\right)^{2}-2 \hat{P} k_{f}-1\right]
\end{aligned}
$$

where $\hat{A}_{p}^{*}=\left(\rho^{*} / 2\right)\left(\rho^{*} \gamma k_{f}-1\right)$ is the inviscid cellular boundary in the far outer wave-number regime given by the last of equations 28. The first of equations 63 thus recovers the cellular stability boundary, but because $i \omega_{0}$ is purely imaginary for $A_{p}^{*}<\hat{A}_{p}^{*}$, stability in that region is determined by the real part of the next nontrivial coefficient in the above expansion for $i \omega$. Thus, setting $i \omega_{2}=0$ in the last of equations 63, the pulsating stability boundary in the far outer wave-number regime is given by

$$
A_{p}^{*} \sim-\rho^{*}\left(1+2 \hat{P} k_{f}\right)^{1 / 2}
$$

which, in the limit $k_{f} \rightarrow 0$, matches with the leadingorder pulsating boundary $A_{p}^{*} \sim-\rho^{*}$ in the outer wave-number region, which is unaffected by viscosity to this order of approximation. Thus, equation 64 , which in terms of $k$ is given by $A_{p}^{*} \sim-\rho^{*}(1+$ $2 \hat{P} \varepsilon k)^{1 / 2}$, is valid for arbitrary wave numbers. Writing $\hat{P}$ in terms of its unscaled counterpart $P$, this expression becomes identical to equation 59 , which thus remains valid in the limit of small $P$.

$$
\text { Acknowledgments }
$$

This work was supported by the U.S. Department of Energy under Contract DE-AC04-94AL85000 and by the NASA Microgravity Science Research Program under Contract C-32031-E.

\section{REFERENCES}

1. Landau, L. D., "On the Theory of Slow Combustion," Acta Physicochim. URSS 19:77-85 (1944); Zh. Eksp. Teor. Fiz. 14:240 (1944).

2. Vosen, S. R., in Twenty-Second Symposium (Intemational) on Combustion, The Combustion Institute, Pittsburgh, 1989, pp. 1817-1825.

3. Levich, V. G., Dokl. Akad. Nauk SSSR 109:975-978 (1956).

4. Armstrong, R. C. and Margolis, S. B., in Twenty-Second Symposium (International) on Combistion, The Combustion Institute, Pittsburgh, 1989, pp. 1807-1815.

5. Armstrong, R. C. and Margolis, S. B., Combust. Flame 77:123-138 (1989).

6. Margolis, S. B., Combust. Flame 113:406-423 (1998).

7. Williams, F. A., Combustion Theory, 2nd ed., Benjamin/ Cumnings, Menlo Park, CA, 1985.

8. Bechtold, J. K. and Margolis, S. B., SIAM J. Appl. Math. 51:1356-1379 (1991). 